\title{
Mathematical modeling and parametric identification of dynamic properties of mechanical subsystems tool and workpiece in turning process
}

\author{
Pham Dinh Tung* \\ Department "Aerospace technology and equipment", Le Quy Don Technical University, Hanoi, \\ Vietnam
}

\begin{abstract}
Research and modeling of dynamic structures of the subsystems tool and the workpiece is given continued attention. This is due to the fact that the analysis of the stability of the cutting process and the self-oscillation is necessary, first of all, to have a model of the dynamics of interacting subsystems tool and workpiece through the cutting process. A similar problem is in the study of the machining accuracy, particularly in cases where the workpiece has significant deformation displacement varying along the trajectory of the tool relative to the workpiece. This paper considers the problem of mathematical modeling and identification of inertial, damping, and stiffness properties of the subsystems tool and workpiece in the dynamics exercises of the cutting process. The algorithms and the results of the identification of parameters in mathematical models of mechanical subsystems tool and workpiece for the case of turning are resulted.
\end{abstract}

\section{Introduction}

Problems of dynamics of cutting process were a subject of researches of many authors carried out within the last 50-60 years. In researches of dynamics of processing on metalcutting machines the main attention was paid to studying of stability of cutting process, and also conditions and mechanisms of excitation of self-oscillations [1-32]. In all these studies, when studying dynamics of cutting process dynamic subsystems of the tool and workpiece without cutting process in linear representation and in mobile system of coordinates, movement of which is defined by trajectories of operative elements of the machine are considered. Subsystems of the tool and workpiece interact through the dynamic coupling formed by cutting process. Dynamic coupling represents model of cutting forces in coordinates of system state, in which following problems are considered: nonlinear dependence of cutting forces on coordinates [5,7,8,15,21-27,30-32]; delay of change of cutting forces relative to change of the movement of system [11-18,21-32]; periodic changes of parameters of dynamic system [16-19]; regularities of formation of new

\footnotetext{
*Corresponding author: phamdinhtung@mail.ru
} 
surface [10]. The questions of modeling and identification of the cutting forces are well described in the works [33-36].

For disclosure of conditions of loss of stability of cutting process, and also conditions of formation of various invariant varieties (asymptotically steady limit cycles, invariant tor, chaotic attractors) researchers offered various models of dynamics of cutting process. In works [7,14-18,22-25] these models are considered in scalar representation. In works [813,19,20,26-32] models are considered in the plane, normal to rotation axis of workpiece, without disclosure of mechanisms of formation of deformation shifts. In this case it is impossible to study many important mechanisms of loss of stability of balance, and also to find out features of transformation of trajectories of the movement of operative elements to trajectories of form-building movements. In addition, in these studies the problem of modeling and identification of dynamic structure subsystems tool and workpiece without cutting process is insufficiently studied.

In article representations of dynamic cutting process stated above are developed, and the main attention to problems of creation of spatial dynamic models and their parametrical identification is paid that significantly supplements known properties of transformative system of cutting process.

\section{Justification of mathematical model and its properties}

When analyzing the dynamics of the cutting process used finite spatial models, leading to the need for analysis of the following differential equation

$$
m(X) \frac{d^{2} X}{d t^{2}}+c(X) \frac{d X}{d t}+k(X) X=F\left(X, f_{c}, t_{c}\right)+f(t)
$$

where $F=\left\{F_{1}\left(X, f_{c}, t_{c}\right), F_{2}\left(X, f_{c}, t_{c}\right), \ldots, F_{6}\left(X, f_{c}, t_{c}\right)\right\}^{T}$ - vector- function of the dynamic characteristics of the cutting process, revealing the dependence of the cutting forces on elastic deformation shifts of the tool and workpiece, and also from cutting conditions: feed per revolution $f_{c}$ and depth of cut $t_{c}$ at specified cutting speed $V_{c} ; X=\left\{X_{1}, X_{2}, \ldots, X_{6}\right\}^{T}-$ Vector elastic deformation shifts of the cutting tool top (first three coordinates) and workpiece (last three coordinates);

$f(t)=\left\{f_{1}(t), f_{2}(t), \ldots, f_{6}(t)\right\}^{T}$ - Components of the cutting forces, cannot be explained in the coordinates of the elastic deformation displacements, are interpreted as noise; $m(X)=\left\lfloor m_{i j}(X)\right\rfloor, \quad c(X)=\left\lfloor c_{i j}(X)\right\rfloor, \quad k(X)=\left\lfloor k_{i j}(X)\right\rfloor, \quad i, j=1,2, \ldots 6 \quad$ - respectively functional matrixes of inertial, dissipative and stiffness coefficients.

In all cases, here and below, the symbol $\{\ldots\}^{T}-$ is the operation of transposition. In system (1), the cutting conditions are subordinate importance, because in the established state they set constant values of forces to which correspond equilibrium points $X^{*}$, the dynamic of the system is studied in variations relative to the equilibrium points. We will focus on the properties of matrices $m, c, k$ in variations relative to the equilibrium point.

In the beginning, we analyze properties of the matrix $k(X)=\left\lfloor k_{i j}(X)\right\rfloor$, which characterizes communication coefficients between variations of external forces and the corresponding established deformation displacements. We will independently consider tool and workpiece subsystems. In the beginning, we will consider the most difficult subsystem of the cutting tool.

V.A Kudinov, J. Tlusty and L. Polacek [4,5] independently of each other showed that the dynamical system support group in low-frequency range can be represented as a 
generalized mass, which is suspended from the non-deformable elements of the loadcarrying system in the form of spatially arranged elastic-dissipative suspensions in an orthogonal coordinate system $0 \gamma_{1} \gamma_{2} \gamma_{3}$. This conclusion was based on the study of the tool deformation under the action of force, orientation of which is changed in space. It has been found that there are collinear directions of the application of external force, which correspond to the elastic deformation of the same direction. These are the axes $\gamma_{1}, \gamma_{2}, \gamma_{3}$. Proceeding from the principles of mechanics these axes are main [37, 38] and they pass through the center of mass of the reduced elastic system. When force is applied in other directions except the axes $\gamma_{1}, \gamma_{2}, \gamma_{3}$ there are collinear and orthogonal components of deformations which always exist at a choice of other system of coordinates, for example of system $0 X_{1} X_{2} X_{3}$.

Modeling of elastic deformation displacement in the linearized form, i.e. in the form of the stiffness matrix, allows us to represent the elastic deformation displacement in the new coordinate system $0 \gamma_{1} \gamma_{2} \gamma_{3},\left\{\gamma_{1}, \gamma_{2}, \gamma_{3}\right\} \in \mathfrak{R}_{\gamma}$ (Fig.1). This coordinate system can be received on the basis of rotation of coordinate system $0 X_{1} X_{2} X_{3},\left\{X_{1}, X_{2}, X_{3}\right\} \in \Re_{X}$ by means of Euler's angles. Stiffness matrix is diagonal in the space $\mathfrak{R}_{\gamma}$. For transition from the coordinate space $\mathfrak{R}_{X}$ to coordinate space $\mathfrak{R}_{\gamma}$, we will execute three consecutive positive rotations on Euler's angles $\varphi, \theta, \psi$. As a result, we will receive a transformation matrix (or a transition matrix) in a form

$$
\Lambda=\left[\begin{array}{ccc}
\cos \varphi \cos \psi-\sin \varphi \cos \theta \sin \psi & -\cos \varphi \sin \psi-\sin \varphi \cos \theta \cos \psi & \sin \varphi \sin \theta \\
\sin \varphi \cos \psi+\cos \varphi \cos \theta \sin \psi & -\sin \varphi \sin \psi+\cos \varphi \cos \theta \cos \psi & -\cos \varphi \sin \theta \\
\sin \theta \sin \psi & \sin \theta \cos \psi & \cos \theta
\end{array}\right]
$$

We note some properties of the matrix $\Lambda: 1 . \mathrm{T}=\Lambda^{-1}$ - transition matrix of coordinate space $\mathfrak{R}_{\gamma}$ to coordinate space $\mathfrak{R}_{X} ; 2 . \Lambda^{T} \Lambda=E$ - single matrix, therefore matrix $\Lambda$ orthogonally. Similar property is fair for a matrix $\mathrm{T}$. Then

$$
X=\Lambda \gamma, \Delta F=\Lambda \Delta F_{\gamma} \text { or } \gamma=\mathrm{T} X, \Delta F_{\gamma}=\mathrm{T} \Delta F
$$

where $\Delta F, \Delta F_{\gamma}$ - vector variations of forces. Therefore

$$
k_{\gamma} \gamma=\Delta F_{\gamma}, \quad k_{\gamma}=\Lambda^{-1} k \Lambda
$$

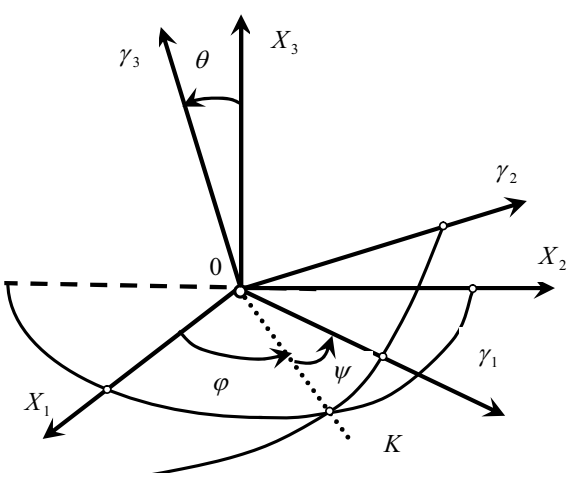

Fig.1. Coordinate system in which the elastic deformation displacement and external forces reports

If for small elastic deformation displacement in variations about equilibrium point the axes $\gamma_{1}, \gamma_{2}, \gamma_{3}$ can be considered major, then the force, having direction coincident with 
one of the axes $\gamma_{1}, \gamma_{2}, \gamma_{3}$, causes elastic deformation displacement only in this direction. In this case, matrix of elastic deformation displacement $k_{\gamma}$ is diagonal.

Thus, the problem is that it is necessary to select the Euler angles so as the matrix $k_{\gamma}$ to be diagonal. It can be done on the basis of the solution of the equations $k_{i j}^{\gamma}(\varphi, \theta, \psi)=0, i \neq j$.

However, this procedure for a spatial case represents great difficulties as the received not diagonal elements of a matrix $k_{\gamma}$ are complex functions of sins and cosines of Euler angles.

We emphasize that the stiffness matrix $k$ in static is a real, positive and symmetric matrix. We note two fundamental properties of symmetric matrices. 1. Eigenvalues of a real, positive and symmetric matrix are positive and real. 2. Eigenvectors corresponding different eigenvalues are orthogonal.

It is known $[39,40]$ that for any real symmetric matrix $c$, there exists an orthogonal matrix $\Lambda$ so as $\Lambda^{T} k \Lambda=k_{\gamma}$, where $k_{\gamma}=\operatorname{diag}\left(k_{\lambda_{1}}, k_{\lambda_{2}}, k_{\lambda_{3}}\right)$ - diagonal matrix whose diagonal elements are the eigenvalues of the matrix $k$. Thus, it is possible to transform a symmetric stiffness matrix $k$ to a diagonal form, and to calculate transformation matrix based on the definition of its eigenvalues and corresponding to them eigenvectors. The stiffness matrix $k_{\gamma}$ in the new coordinate system has the diagonal elements being the eigenvalues of the matrix $k$.

For the considered problem the most important case is when the matrix $k$ has distinct eigenvalues. Then one has:

$$
\operatorname{det}(k-\lambda E)=0
$$

For each eigenvalue $\lambda_{i}$ we define eigenvector $g^{i}=\left(g_{i 1}, g_{i 2}, g_{i 3}\right)^{T}$ which satisfies the system of equations

$$
\left(c-\lambda_{i} E\right) g^{i}=0
$$

As noted above, the eigenvectors $g^{1}, g^{2}, g^{3}$, corresponding to different eigenvalues $\lambda_{1}, \lambda_{2}, \lambda_{3}$, are mutually orthogonal. We will find normalized eigenvectors $e^{1}, e^{2}, e^{3}$ corresponding to vectors $g^{1}, g^{2}, g^{3}$ under condition $\left(e^{i}, e^{i}\right)=1, i=1,2,3$.

$$
e^{i}=\frac{g^{i}}{\left\|g^{i}\right\|}
$$

When the orthogonal transformation matrix $\Lambda$ whose columns are normalized eigenvectors $e^{i}$,

$$
\Lambda=\left(e^{1}, e^{2}, e^{3}\right)=\left[\begin{array}{lll}
e_{11} & e_{21} & e_{31} \\
e_{12} & e_{22} & e_{32} \\
e_{13} & e_{23} & e_{33}
\end{array}\right]
$$

Matrix (8) can be considered as a matrix of angular transformation coefficients angular of space coordinate $\mathrm{X}$ to $\mathrm{Y}$. Now we can define the Euler angles. 


$$
\theta=\arccos e_{33} ; \varphi=\arcsin \left(\frac{e_{31}}{\sqrt{1-\left(e_{33}\right)^{2}}}\right) ; \psi=\arcsin \left(\frac{e_{13}}{\sqrt{1-\left(e_{33}\right)^{2}}}\right)
$$

A similar algorithm can be used to determine the deformation properties of the workpiece subsystem. In this case, it can be assumed that the deformation displacement of the workpiece in the feed rate direction does not exist. Then, the deformation model can be studied in a plane normal to the axis of rotation of the workpiece. In this case, the ellipsoid of stiffness for the spatial case is converted into ellipse of stiffness in the plane (Fig. 2). Then we can immediately obtain the expression for the orientation of the ellipse of stiffness and values of its diagonal elements. If the matrix of elastic deformation displacement $k$ in the space $\mathfrak{R}_{X}$ are givens placement space, their expression $k_{\gamma}$ in space $\mathfrak{R}_{\gamma}$ is obtained based on the following transformation:

$$
k_{\gamma}=\mathrm{A}_{p}^{-1} c \Lambda_{p}
$$

Where $\Lambda_{p}=\left[\begin{array}{cc}\cos \alpha & \sin \alpha \\ -\sin \alpha & \cos \alpha\end{array}\right]$ - transition matrix of system coordinates in plane.

For this matrix, we perform the coordinate rotation transformation

$$
\begin{aligned}
& k_{\gamma}=\left[\begin{array}{cc}
\cos \alpha & -\sin \alpha \\
\sin \alpha & \cos \alpha
\end{array}\right] \cdot\left[\begin{array}{ll}
k_{11} & k_{21} \\
k_{12} & k_{22}
\end{array}\right] \cdot\left[\begin{array}{cc}
\cos \alpha & \sin \alpha \\
-\sin \alpha & \cos \alpha
\end{array}\right]= \\
& =\left[\begin{array}{cc}
k_{11} \cos ^{2} \alpha+k_{22} \sin ^{2} \alpha-2 k_{12} \sin \alpha \cos \alpha & \left(k_{22}-k_{11}\right) \sin 2 \alpha+2 k_{12} \cos 2 \alpha \\
\left(k_{22}-k_{11}\right) \sin 2 \alpha+2 k_{12} \cos 2 \alpha & k_{11} \sin ^{2} \alpha+k_{22} \cos ^{2} \alpha+2 k_{12} \sin \alpha \cos \alpha
\end{array}\right] .
\end{aligned}
$$

It is required that off-diagonal elements in the matrix (11) are equal to zero. $\left(k_{22}-k_{11}\right) \sin 2 \alpha+2 k_{12} \cos 2 \alpha=0$, then we obtain

$$
\alpha=\frac{1}{2} \operatorname{arctg}\left(\frac{2 k_{12}}{\left(k_{11}-k_{22}\right)}\right)
$$

Matrix $k_{\gamma}$ is diagonal, that is to say:

$$
k_{\gamma}=\operatorname{diag}\left(k_{01}, k_{02}\right)
$$

where $k_{01}=\left(k_{11} \cos ^{2} \alpha+k_{22} \sin ^{2} \alpha-2 k_{12} \sin \alpha \cos \alpha\right) ; k_{02}=\left(k_{11} \sin ^{2} \alpha+k_{22} \cos ^{2} \alpha+2 k_{12} \sin \alpha \cos \alpha\right)$. Taking the inverse coordinate transformation $\left\{\gamma_{1}, \gamma_{2}\right\}^{T}$ into $X=\left\{X_{1}, X_{2}\right\}^{T}$, we obtain expression to determine the matrix of deformation displacement of the tool tip for a given matrix (13)

$k=\left[\begin{array}{cc}\cos \alpha & \sin \alpha \\ -\sin \alpha & \cos \alpha\end{array}\right] \cdot\left[\begin{array}{cc}k_{01} & 0 \\ 0 & k_{02}\end{array}\right] \cdot\left[\begin{array}{cc}\cos \alpha & -\sin \alpha \\ \sin \alpha & \cos \alpha\end{array}\right]==\left[\begin{array}{cc}k_{01} \cos ^{2} \alpha+k_{02} \sin ^{2} \alpha & \frac{1}{2}\left(k_{02}-k_{01}\right) \sin 2 \alpha \\ \frac{1}{2}\left(k_{02}-k_{01}\right) \sin 2 \alpha & k_{01} \sin ^{2} \alpha+k_{02} \cos ^{2} \alpha\end{array}\right]$.

When $k_{01}=k_{02}$ matrix $k$ is diagonal. This case is typical for the workpiece subsystem. For this case, any orthogonal coordinate system will be characterized by the fact that force in any direction causes the deformation displacement of the same direction. This is natural, since the workpiece subsystem has symmetrical deformation properties. Therefore, when 
modeling deformation properties of the workpiece subsystem scalar representation can be used.

Studying of static and dynamic deformation properties of a subsystem of the cutting tool, including the correlation spectral analysis of reactions of system on external delta-like disturbances, shows that in the frequency range limited to the first forms of oscillations, following property holds. Oscillatory reactions of a subsystem of the tool, caused by external delta-like disturbances, as well as static forces, possess property of spatial selectivity. And, both for dynamic and static reactions the directions of axes of the collinear direction of deformation displacements coincide. Therefore for a subsystem of the tool the schematization representing the generalized weight, suspended in three elastic and dissipative suspension brackets is fair. Such schematization in the plane is given in Fig.2.
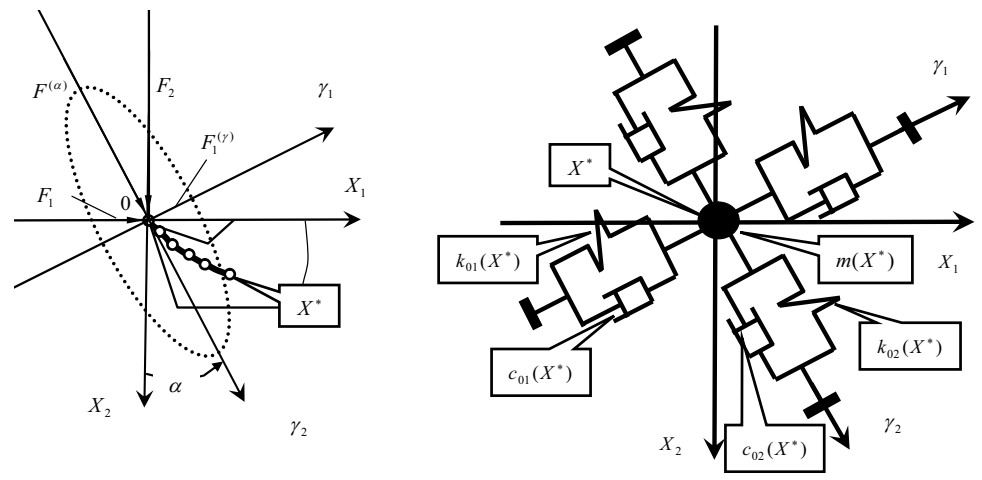

Fig.2. The coordinate system in which the elastic deformation displacement of the tip of cutting tool external forces in plane are measured.

Therefore, the following important for identification procedures properties of the coordinate transformation and parameters of matrices $m, c, k$ are correct. $1 . \Lambda^{T} k \Lambda=k_{\gamma}$, $\Lambda^{T} c \Lambda=c_{\gamma}, \quad \Lambda^{T} m \Lambda=m_{\gamma}$. 2. Transformation of the space $\mathfrak{R}_{X}$ into $\mathfrak{R}_{\gamma}$ or $\mathfrak{R}_{\gamma}$ into $\mathfrak{R}_{X}$ is characterized by a linear operation. Consequently, the characteristic polynomials in these spaces will be the same, that means $\Delta(p)=\Delta_{\gamma}(p)$.

\section{Parametric identification}

In space $\mathfrak{R}_{X}$ at invariable on the module force excitations the reactions of elastic displacements, speeds and accelerations describe the ellipsoid which main axes remain same. Therefore diagonal elements of matrixes $m, c, k$ of space $\mathfrak{R}_{\gamma}$, and also elements of matrixes of slopes (8) or Euler's angles (9) are subject to identification. As matrixes of slopes for $m, c, k$ are the same, identification is performed in two stages. At the first stage identification of $k$ is carried out. Giving to forces the following measurable increments $\Delta F^{(1)}=\left\{\Delta F_{1}, 0,0\right\}^{T}, \Delta F^{(2)}=\left\{0, \Delta F_{2}, 0\right\}^{T}, \Delta F^{(3)}=\left\{0,0, \Delta F_{3}\right\}^{T}$ and respectively measuring variations $x^{(1)}=\left\{x_{1}^{(1)}, x_{2}^{(1)}, x_{3}^{(1)}\right\}^{T}, \quad x^{(2)}=\left\{x_{1}^{(2)}, x_{2}^{(2)}, x_{3}^{(2)}\right\}^{T} \quad$ and $\quad x^{(3)}=\left\{x_{1}^{(3)}, x_{2}^{(3)}, x_{3}^{(3)}\right\}^{T} \quad$ of deformation displacements, we obtain systems for estimation of elements of the matrix $k\left(X^{*}\right)$.

$$
x^{(e)} k^{(e)}\left(X^{*}\right)=\Delta F^{(e)}
$$


where $x^{e}=\left[\begin{array}{ccccccccc}x_{1}^{(1)} & x_{2}^{(1)} & x_{3}^{(1)} & 0 & 0 & 0 & 0 & 0 & 0 \\ 0 & 0 & 0 & x_{1}^{(1)} & x_{2}^{(1)} & x_{3}^{(1)} & 0 & 0 & 0 \\ 0 & 0 & 0 & 0 & 0 & 0 & x_{1}^{(1)} & x_{2}^{(1)} & x_{3}^{(1)} \\ x_{1}^{(2)} & x_{2}^{(2)} & x_{3}^{(2)} & 0 & 0 & 0 & 0 & 0 & 0 \\ 0 & 0 & 0 & x_{1}^{(2)} & x_{2}^{(2)} & x_{3}^{(2)} & 0 & 0 & 0 \\ 0 & 0 & 0 & 0 & 0 & 0 & x_{1}^{(2)} & x_{2}^{(2)} & x_{3}^{(2)} \\ x_{1}^{(3)} & x_{2}^{(3)} & x_{3}^{(3)} & 0 & 0 & 0 & 0 & 0 & 0 \\ 0 & 0 & 0 & x_{1}^{(3)} & x_{2}^{(3)} & x_{3}^{(3)} & 0 & 0 & 0 \\ 0 & 0 & 0 & 0 & 0 & 0 & x_{1}^{(3)} & x_{2}^{(3)} & x_{3}^{(3)}\end{array}\right]$ matrix of experimentally

determined deformation displacements; $\Delta F^{(e)}=\left\{\Delta F_{1}^{(1)}, 0,0,0, \Delta F_{2}^{(2)}, 0,0,0, \Delta F_{3}^{(3)}\right\}^{T}$ - vector of measured external values of forces acting on the cutting tool; $k^{(e)}=\left\{k_{11}, k_{21}, k_{31}, k_{12}, k_{22}, k_{32}, k_{13}, k_{23}, k_{33}\right\}^{T}$ - vector of subjecting to estimation parameters of stiffness matrix. We will note that when carrying out researches deformations are measured by the devices whose base is located on bearing system of the machine. In this case, in the guides.

For the fixed value of a equilibrium point, from (14) we obtain the massif of values $k_{i j}$, $i, j=1,2,3 \quad k_{i j}=k_{j i}$ when $i \neq j$, by which we obtain estimation of stiffness parameters by mathematical expectation and values and their variances.

Mathematical expectations $\hat{k}_{i j}$ are estimations of stiffness matrix in the coordinate system $O X_{1} X_{2} X_{3}$ for given equilibrium point. For them variances $\hat{\sigma}_{i j}$ estimated by the value of the standard deviations are also determined. Matrixes of slopes and values of diagonal elements of stiffness matrix carried out to normal coordinates are determined by the obtained data.

At the second stage parameters of matrixes $m$ and $c$ are estimated. Thus, information on estimations of weight functions of system (oscillatory reactions of system to delta-like disturbances) and their frequency images is used as the initial input. At determination of the generalized masses values of the resonant frequencies close at small damping coefficients to own frequencies of system are the basis. In turn, resonant frequencies are defined from condition of minimum of characteristic polynomial of system $\Delta(s)=\Delta_{\gamma}(s)$ after substitution $s=j \omega$ and calculation of a square of the module, that is $\Delta(j \omega) \Delta(-j \omega)=\Delta_{\gamma}(j \omega) \Delta_{\gamma}(-j \omega)$. In turn, $\Delta_{\gamma}(s)=\prod_{i=1}^{i=3}\left(k_{0 i}+c_{0 i} s+m_{0 i} s^{2}\right)$. Easily measurable autospectrums of oscillatory reactions of system are the basis for estimation of the generalized masses $m_{0 i}$. As $c_{0 i}$ are defined at the first stage of carrying out researches, the generalized masses are estimated by obvious dependences $m_{0 i}=c_{0 i} /\left(\Omega_{0 i}\right)^{2}$, where $\Omega_{0 i}$ resonant frequency of the corresponding conservative system. In this case, one of resonant frequencies is equal to natural frequency.

As oscillatory contours are high-Q, damping coefficient can also be defined based of the analysis of broadening of the spectral line of each of resonant frequencies, that is $\xi_{i}=\left(\Omega_{0 i}^{(+)}-\Omega_{0 i}^{(-)}\right)\left(\Omega_{0 i}\right)^{-1}, i=1,2,3$ where $\left(\Omega_{0 i}^{(+)}-\Omega_{0 i}^{(-)}\right)$- width of resonant curve at the height of amplitudes of 0,707 from resonant amplitude. Then damping coefficients $c_{0 i}$ are equal to:

$$
c_{0 i}=2 \xi_{i} \Omega_{0 i} m_{0 i}=2 \xi_{i} \sqrt{m_{0 i} k_{0 i}}, i=1,2,3 .
$$


Then natural frequencies for nonconservative system are more precisely specified

$$
\Omega_{i}^{(\text {cut })}=\left(\Omega_{0 i}\right)\left[1-2\left(\xi_{i}\right)^{2}\right]^{\frac{1}{2}}, i=1,2,3,
$$

where $\xi$ - attenuation coefficient_of a considered oscillatory contour. Attenuation coefficients in mechanical systems of metal-cutting machines usually lie in the range $(0,02$ 0,06). We will emphasize that for definition $m_{0 i}$ it isn't obligatory to measure revolting forces, as values of the generalized stiffnesses $k_{0 i}$ are given. Frequencies $\Omega_{01}$ and $\Omega_{02}$ are determined based on autospectrums in dimensionless form. It is not neccesary to apply an external force disturbance oriented towards axes of space $\Lambda$ to estimate parameters $k_{0 i}$. Besides we will pay attention to the rule connecting ratios of natural frequencies of system with values of diagonal elements of a matrix of inertia in space $\Omega_{\gamma}$. If ratios $\left(\Omega_{03}\right)^{2} /\left(\Omega_{01}\right)^{2}=k_{01} / k_{03}$ and $\left(\Omega_{03}\right)^{2} /\left(\Omega_{02}\right)^{2}=k_{02} / k_{03}$ are carried out, then $m_{0 i}=$ const. Then in space $\Omega_{X}$ matrix of inertia is also diagonal, and all diagonal elements are equal, $m_{0 i}=$ const . In this case the mass of a suspension bracket of the tool can be considered invariable and independent of a matrix of the slopes characterizing orientation of elastic and dissipative suspension brackets.

Main complexity of parametrical identification of matrixes $c$ and $m$ consists in need of estimation of parameters for various equilibrium points of system. For this purpose it is necessary to create the external force field displacing equilibrium point $\left(X_{1}^{*}, X_{2}^{*}, X_{3}^{*}\right)$. To do this, we conduct an additional elastic element. Additional stifness brings errors, first of all, at determination of natural frequencies. Main requirement to an elastic element - linearity of elastic properties, robustness of bending oscillation and orientation in the direction of axes $\gamma_{i}, i=1,2,3$. thereby its dissipation, in comparison with the dissipation of the cutting tool subsystem, can be ignored. Then introduced additional bond modifies one of the diagonal stiffening elements that allows to evaluate changes in the parameters of the system depending on the displacement of the equilibrium point without changing the general procedure

\section{Analysis of features of dynamic subsystems}

We will analyse features of dynamic subsystems of the tool and workpiece on the example of the lathe $1 \mathrm{~K} 62$. We will be limited to a case when the subsystem of the tool is considered in the plane, normal to an axis of rotation of workpiece. Experimentally determined chart of deformation displacements of top of the tool is submitted in Fig. 3 at two moduleconstant forces having various directions in the plane. Trajectories of deformation displacement of top of the tool are shown on the chart (two point-by-point fatty curves, corresponding to two values module of force of $1000 \mathrm{~N}$ and $2000 \mathrm{~N}$ ). In this case the tool extension from the work holding assembly_is equal $35 \mathrm{~mm}$. Dotted straight lines showed some directions of action of force. Besides the directions of the external force corresponding to axes are considered $X_{1}$ (extraction direction of displacements) and $X_{2}$ (direction of cutting speed). The directions of deformation displacements orthogonal to the direction of action of force are shown by arrows. On illustration only collinear deformation displacements correspond to angles $\alpha=19^{\circ}$ and $\alpha=109^{\circ}$. Both collinear and orthogonal deformation displacements correspond to all other directions. It is important to emphasize that the directions of only collinear deformation displacements do not depend on value of the module of force in the range of its change $(200 \mathrm{~N}-2000 \mathrm{~N})$. 


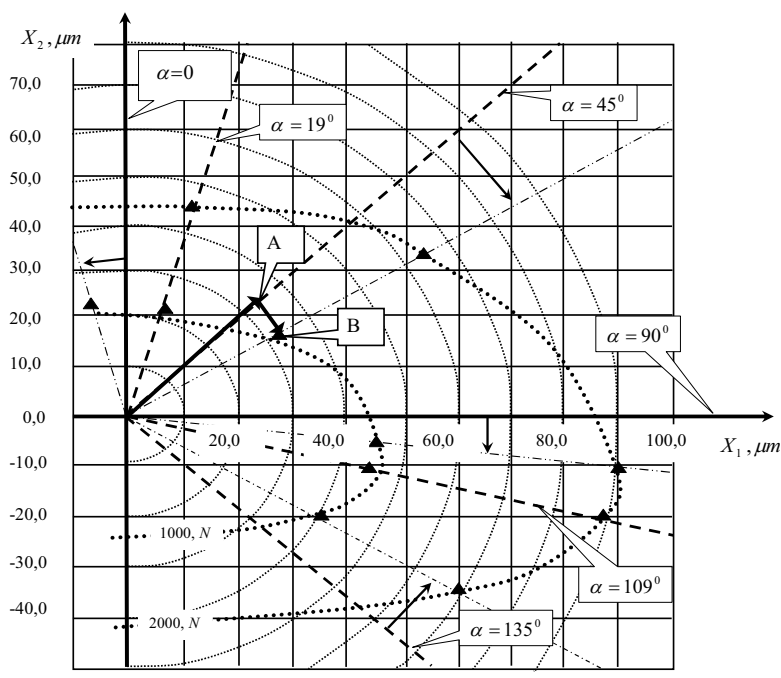

Fig. 3. Typical chart of total deformation displacements of top of the cutting tool depending on the direction and the module of external force for support group of the machine 1K62.

The values of stiffness matrixes change by increasing external forces. Thus, orientation of ellipse of stiffness practically does not change. Dependences of stiffnesses on coordinates $\left\{\gamma_{1}, \gamma_{2}\right\}^{T}$ are shown in Fig. 4. They are well approximated by exponential functions, that is

$$
k_{01}\left(\gamma_{1}\right)=k_{10}^{(1)}+k_{10}^{(2)}\left[1-\exp \left(-\frac{\gamma_{1}}{T_{\gamma}^{(1 k)}}\right)\right] ; k_{02}\left(\gamma_{1}\right)=k_{20}^{(1)}+k_{20}^{(2)}\left[1-\exp \left(-\frac{\gamma_{1}}{T_{\gamma}^{(2 k)}}\right)\right]
$$

where $k_{10}^{(1)}, k_{20}^{(1)}$ - stiffness values at the initial stage of the applying of external loading; $k_{10}^{(2)}, k_{20}^{(2)}$ - values of increment stiffness in process of increasing deformation displacements; $T_{\gamma}^{(1 k)}, T_{\gamma}^{(2 k)}$ - parameters characterizing speed of increase in parameters of stiffness. Stiffness functions have dimension $N / m m$, and $T_{\gamma}^{(1 k)}, T_{\gamma}^{(2 k)}$ have dimension $\mathrm{mm}$.

Almost full compliance of experimental and theoretical results is defined by the fact that parameters of stiffness matrixes in the directions $\left\{\gamma_{1}, \gamma_{2}\right\}$ are determined based on identification.

However, they are determined in the analysis of deformation displacements by the directions $\left\{F_{1}, 0\right\} \quad$ и $\left\{0, F_{2}\right\}$, and deformation displacements are calculated on the algorithms stated above. The greatest error, which does not exceed $10 \%$, is appeared in area of small force loading of top of the cutting tool by external forces. With the big forces (more than $500 \mathrm{~N}$ ) the error does not exceed $(1,0-2,0) \%$. We will provide also data on the key parameters characterizing deformation properties of top of the cutting tool for various machines of turning group (Tab. 1). Cross section of the tool is defined by the following sizes: base width $-30 \mathrm{~mm}$, height $-26 \mathrm{~mm}$. Length is specified in Table 1 . 

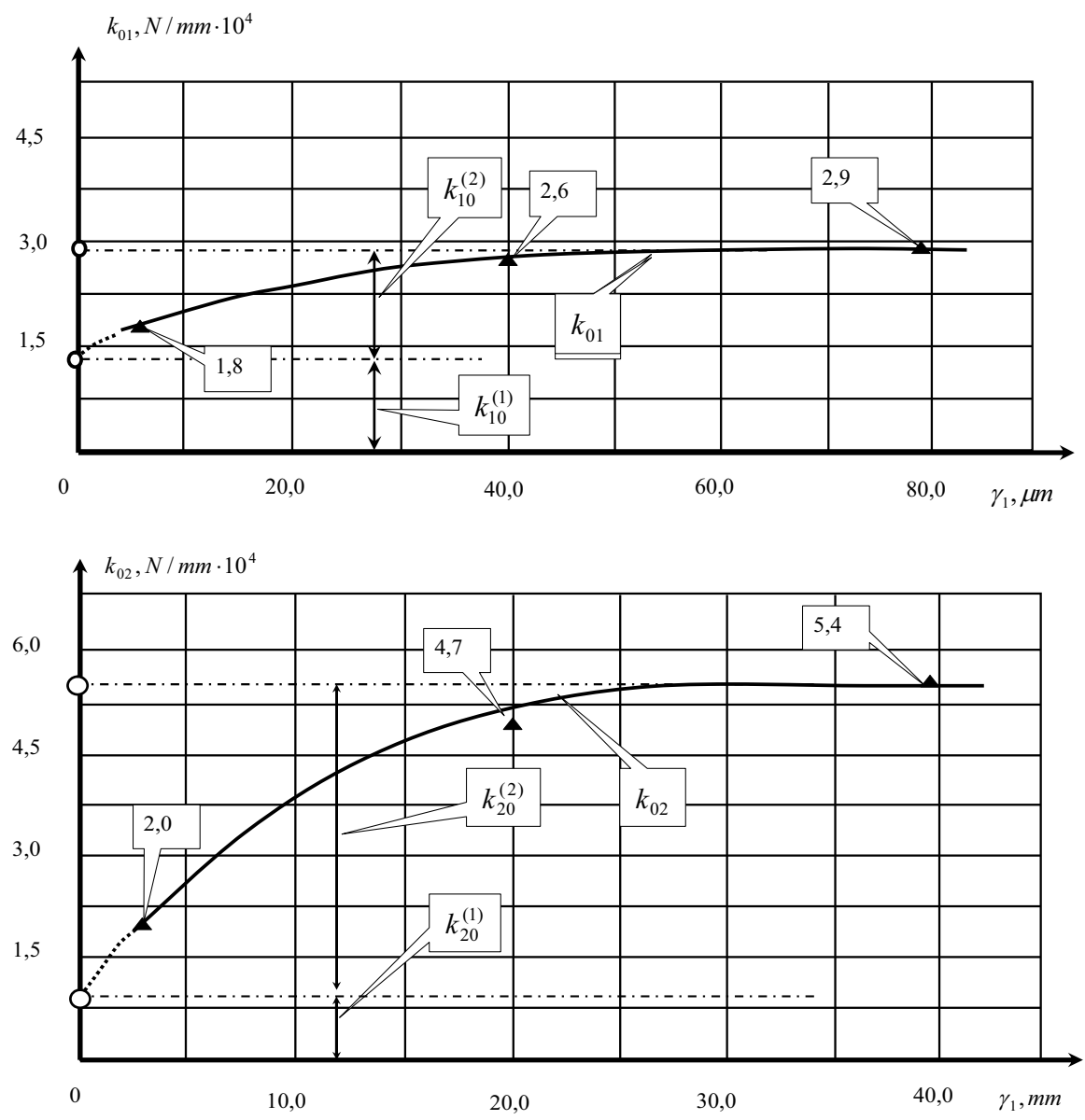

Fig. 4. Dependence of stiffness matrixes elements of deformation displacements of the tool tip in the directions of orientation of stiffness ellipses depending on deformation displacements in the same directions

These tables show that the parameters characterizing deformation displacements depend not only on machine type, but also on parameters of the cutting tool, in particular, on its departure from the work holding assembly. Thus, it is not difficult to show that increase in elastic deformation displacements of top of the cutting tool in direction $X_{2}$ increases significantly more than the deformation of the tool shank. Here we have to consider the fact that as the results of increasing departure of the tool the moment of rotation turning all support group increases, and also redistribution of contact stresses and deformations in points of interface of the separate details entering into support group. It explains the dependence of orientation of axes of an stiffness ellipse depending on external forces at big departures of the tool from the tightening adaptation, setting equilibrium point of tool system. Orientation of these axes influences area of stability of dynamic cutting system considered in variations about equilibrium point. 
Table 1. Parameters of stiffness matrixes of deformation displacements for various machines and tool extension from the workholding assembly

\begin{tabular}{|c|c|c|c|c|}
\hline $\begin{array}{c}\text { Russian machine } \\
\text { type }\end{array}$ & $k_{01}, N / m m$ & $k_{02}, N / m m$ & $\alpha^{0}$ & $\begin{array}{c}\text { tool extension } \\
l, m m\end{array}$ \\
\hline 1 K62 & $2,9 \cdot 10^{4}$ & $5,4 \cdot 10^{4}$ & $10^{0}$ & 35 \\
\hline 1 K62 & $0,7 \cdot 10^{3}$ & $5,1 \cdot 10^{4}$ & $\left(10^{0}-18^{0}\right)$ & 120 \\
\hline УT16Ф3 & $4,3 \cdot 10^{4}$ & $6,7 \cdot 10^{4}$ & $12^{0}$ & 35 \\
\hline 16 К20 NC210 & $3,7 \cdot 10^{4}$ & $6,5 \cdot 10^{4}$ & $14^{0}$ & 54 \\
\hline $1 \mathrm{~B} 340 \Phi 30$ & $2,8 \cdot 10^{4}$ & $4,5 \cdot 10^{4}$ & $19^{0}$ & 54 \\
\hline
\end{tabular}

As for a damping matrix, it possesses such properties: orientation of axes of the collinear directions of ellipse of dissipation coincides with orientation of axes of an stiffness ellipse. Nevertheless the damping coefficient in the direction of bigger stiffness is less, than in the direction of smaller stiffness. Besides a manifested tendency is always detected. In process of increasing displacement of the equilibrium point depending on external force, dissipative influence of support group on oscilation decreases. Here, the approximation similar to (18) is also possible

$$
c_{01}\left(\gamma_{1}\right)=c_{10}^{(1)}+c_{10}^{(2)} \exp \left(-\frac{\gamma_{1}}{T_{\gamma}^{(1 c)}}\right) ; c_{02}\left(\gamma_{1}\right)=c_{20}^{(1)}+c_{20}^{(2)} \exp \left(-\frac{\gamma_{1}}{T_{\gamma}^{(2 c)}}\right),
$$

where $\left(c_{10}^{(1)}+c_{10}^{(2)}\right),\left(c_{20}^{(1)}+c_{20}^{(2)}\right)$ - values of damping coefficients at the initial stage of applying external load; $c_{10}^{(2)}, c_{20}^{(2)}$ - values of increment of damping coefficients in process of increasing deformation displacements; $T_{\gamma}^{(1 c)}, T_{\gamma}^{(2 c)}$ - parameters characterizing speed of reduction of damping coefficients. Damping coefficients have dimension $\mathrm{N} \cdot \mathrm{s} / \mathrm{mm}$, $T_{\gamma}^{(1 c)}, T_{\gamma}^{(2 c)}$ have dimension $\mathrm{mm}$.

In process of increasing external forces value of the generalized weight changes also, but it remain invariable in the directions. Therefore when modeling dynamic tool subsystem the schematization in the form of some constant generalized weight suspended in space on two orthogonal elastic dissipative suspension brackets is fair. We will notice that such schematization is fair in the frequency range to (4.0-5.0) KHz.

As for a workpiece subsystem, how it is already noted, its properties are representable in the considered frequency range in the form of generalized weight suspended in space on elastic and dissipative suspension brackets, having equal values of stiffnesses and damping coefficients. Elastic properties of workpiece subsystem depend on the coordinate of movement of contact point of the tool about workpiece. Thus natural frequencies of oscillation of workpiece subsystem remain invariable. For agreement of this contradiction, it is necessary to consider in addition change of the specified weight along trajectory of movement of the tool about workpiece (fig. 5). 


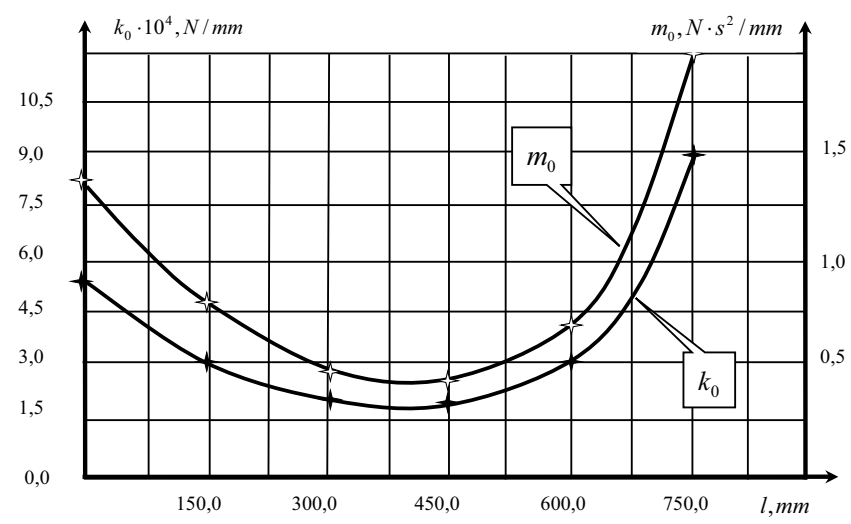

Fig. 5. Changes of the specified coefficients of stiffness and weight in dependences on coordinate $l$ along axis of rotation of workpiece

\section{Conclusion}

The reduced mathematical models of the elastic deformation properties of the subsystems of the cutting tool and workpiece allow us to consider the principal features of the dynamics of the deformation displacement of the cutting tool top and workpiece at the contact point between cutting tool and workpiece. In this moment the subsystem cutting tool in the frequency range up to $5,0 \mathrm{kHz}$, it is possible to allocate three orthogonal directions, deformations on which have only collinear components. For these axes, stiffness and damping matrices are diagonal. These matrices are symmetric and positive definite in any other orthogonal coordinates system. The elements of these matrices are dependent on the matrices of the angular coefficients or Euler angles. For the analysis of deformation displacement of the workpiece subsystem, enough to have information on distribution of scalar size of rigidity on length, added with data on change of inertial coefficient.

The reduced models and methods of its parametrical identification significantly supplement known concepts of elastic properties of interacting subsystems of the tool and workpiece through the dynamic coupling formed by cutting process, as in a solution of the problem of transformation of executive elements movement trajectory in a form-building movements trajectory of the tool relative to workpiece, as well as and in the stability analysis of these trajectories.

The given formalism and technique of parametrical identification can be equally widespread on other technological processing on metal-cutting machines.

\section{References}

1. S. A. Tobias, Machine Tool Vibrations, (Blackie, London, 1965)

2. H.E. Merritt, Trans. Am. Soc. Mech. Engns, 87, 447 (1965)

3. I. Koenigsberger, J. Tlusty, Structure of Machine Tools (Pergamon Press, Oxford, 1971)

4. J.Tlusty, A. Polacek, C. Danek, J. Spacek. Selbsterregte Schwingungen an Werkzeugmaschinen (VEB Verlag Technik, Berlin, 1962)

5. V.A. Kudinov, Dynamics of machine tools (In Russian) (Publishing house "Engineering", Moscow, 1967) 
6. S. Kato and Marui, Trans. ASME J. Engns Ind., 96, 179 (1974)

7. S. Lin and, C. I. Weng, Int. J. Mach. Tools Manufact., 30, 53 (1990)

8. I. Grabec, J. Mach. Tools Manufact, 28, 19 (1988)

9. Y. Altintas, and M. Weck, CIRP Annals-Manufacturing Technology, 53(2), 619 (2004)

10. M. Wan, Y. Altintas,. International Journal of Machine Tools and Manufacture, 87, 16 (2014)

11. Y. Kurata, S.D. Merdol, Y. Altintas, N. Suzuki, E. Shamoto, Journal of advanced mechanical design, Systems, and Manufacturing, 4 1107, (2010)

12. M. Eynian, Y. Altintas, Journal of Manufacturing Science and Engineering, 132, 1 (2010)

13. Y. Altintas, G. Stepan, D. Merdol, Z. Dombovari, CIRP-Journal of Manufacturing Science and Technology, 1, 35,. (2008)

14. G. Stepan, Philosophical Transactions of the Royal Society of London, A359, 739 (2001)

15. T. Insperger, G. Stepan, Periodica Polytechnica, 44, 47 (2000)

16. T. Insperger, G. Stepan, International Journal for Numerical Methods in Engineering 61, 117 (2004)

17. G. Stepan et al. Delay, International Journal of Bifurcation and Chaos, 15, 9, 2783 (2005)

18. Z. Yao, D. Mei, Z. Chen, Journal of Sound and Vibration, 330 (13), 2995 (2011)

19. Z V. L.akovorotny, D.T. Pham, T.H. Pham, Bulletin DSTU, 5-6 (74-75), 97 (2013) (In Russian)

20. S.M. Afazov, S.M. Ratchev, J. Segal, A.A. Popov, International Journal of Machine Tools \& Manufacture, 56, 28 (2012)

21. M. Wiercigroch, A. D.-H. Cheng, Chaos, Solitons and Fractals, 8, 715 (1997)

22. G. Litak, Chaos, Solitons \& Fractals, 13, 1531-5 (2002)

23. F. C. Moon and T. Kalm'ar-Nagy, Phil. Trans. R. Soc. Lond. A 359, 695 (2001)

24. R R.usinek, A. Weremczuk, J. Warminski, Mechanics and Mechanical Engineering. 15, 4, 131 (2011)

25. B. Balachnadran, Phil Trans The Royal Society of London A Mathematical Physical And Engineering Science, 359 (1781), 793 (2001)

26. M.S. Fofana, Chaos Solitons and Fractals, 17, 731-47 (2003)

27. A. Otto, S. Rauh, M.Kolouch, G. Radons, INT J MACH TOOL MANU, 82-83, 50-58 (2014)

28. L. Vela-Martínez, J.Carlos Jáuregui-Correa, E. Rubio-Cerda, G. Herrera-Ruiz, A. Lozano-Guzmán, INT J MACH TOOL MANU, 48, 9, 1054 (2008)

29. G. Urbikain, A. Fernández, L.N. López de Lacalle, M.E. Gutiérrez, INT J MACH TOOL MANU, 67, 35 (2013)

30. J.G. Kabaldin. Self-organization and nonlinear dynamics in the processes of friction and wearout of the tool during cutting (Publishing center of Komsomolsk on Amur state technical University, 2003) (In Russian)

31. L.S. Murashkin, S.L. Murashkin. Applied nonlinear mechanics of metal cutting machines (Publishing house "Engineering", Leningrad, 1977) (In Russian) 
32. V.L. Zakovorotny, A.D. Lukyanov, Dong Anh Nguyen, Pham Dinh Tung, Synergetic system controlled synthesis of dynamics of machine tools, taking into account the evolution of relations. (Publishing House DSTU, Rostov-on-Don, 2008) (In Russian)

33. G. Stepan, Z. Dombovari, J. Munoa, CIRP Annals - Manufacturing Technology, 60 (1), 113 (2011)

34. Y. Altintas, M. Eynian, H. Onozuka, CIRP Annals-Manufacturing Technology, 57, 371 (2008)

35. E. Budak, L.T. Tunc, CIRP Annals-Manufacturing Technology, 59, 403 (2010)

36. B. Powałka, M. Pajor, S. Berczyński, Advances in manufacturing science and technology, 33, 3 (2009)

37. E.N. Berezkin, Lectures on theoretical mechanics. (MGU, Moscow, 1968) (In Russian)

38. N.N. Buchholz, The main course of theoretical mechanics. H 11. (Science, Moscow, 1972) (In Russian)

39. R. Horn, Ch. Johnson, Matrix analysis (Mir, Moscow, 1989) (In Russian)

40. F.R. Gantmakher, Lectures by analytical mechanics (Science, Moscow, 1966) (In Russian) 